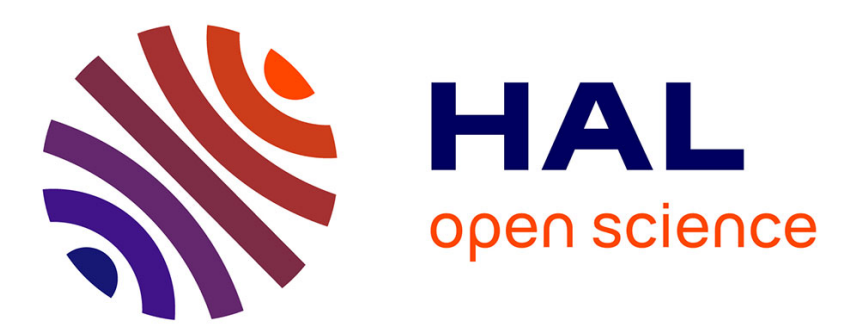

\title{
A new description of scapulothoracic motion during arm movements in healthy subjects.
}

Alexandra Roren, Marie Martine Lefèvre-Colau, Serge Poiraudeau, Fouad Fayad, Viviane Pasqui, Agnès Roby-Brami

\section{- To cite this version:}

Alexandra Roren, Marie Martine Lefèvre-Colau, Serge Poiraudeau, Fouad Fayad, Viviane Pasqui, et al. A new description of scapulothoracic motion during arm movements in healthy subjects.. Manual Therapy, 2014, 20 (1), pp.46-55. 10.1016/j.math.2014.06.006 . hal-01050758

\section{HAL Id: hal-01050758 https://hal.science/hal-01050758}

Submitted on 25 Jul 2014

HAL is a multi-disciplinary open access archive for the deposit and dissemination of scientific research documents, whether they are published or not. The documents may come from teaching and research institutions in France or abroad, or from public or private research centers.
L'archive ouverte pluridisciplinaire HAL, est destinée au dépôt et à la diffusion de documents scientifiques de niveau recherche, publiés ou non, émanant des établissements d'enseignement et de recherche français ou étrangers, des laboratoires publics ou privés. 


\title{
A new description of scapulothoracic motion during arm movements in healthy subjects
}

\author{
Published in Manual Therapy
}

DOI: 10.1016/j.math.2014.06.006

Alexandra Roren ${ }^{\mathrm{a},}$, Marie-Martine Lefevre-Colau ${ }^{\mathrm{a}}$, Serge Poiraudeau $^{\mathrm{a}, \mathrm{f}}$, Fouad Fayad $^{\mathrm{b}}$, Viviane Pasqui ${ }^{\mathrm{c}, \mathrm{d}, \mathrm{e}}$, Agnès Roby-Brami $^{\mathrm{a}, \mathrm{c}, \mathrm{d}, \mathrm{e}, \mathrm{f}, \text {, }}$

a: Department of Physical Medicine and Rehabilitation, Cochin Hospital (AP-HP), Paris Descartes University, Paris, France ${ }^{b}$

b: Department of Rheumatology, Hotel-Dieu de France Hospital, Saint-Joseph University, Beirut, Lebanon

c: ISIR (Institute of Intelligent Systems and Robotics), CNRS UMR 7222, Paris, France

d: Sorbonne Universités, UPMC University Paris 06, Paris, France

e ISIR-AGATHE, INSERM U 1150, Paris, France

f Institut Fédératif de Recherche sur le Handicap, INSERM, Paris, France 


\section{INTRODUCTION}

A sound understanding of the kinematics of the shoulder complex is important for the clinical evaluation and treatment of many pathological conditions (Sahrmann, 2001). It has long been observed clinically that the scapula over the thorax is finely coordinated with the rotations which occur at the glenohumeral joint ("scapulo-humeral rhythm") (Codman, 1934). During scapulothoracic motion, there are simultaneous rotations of the clavicle at the sternoclavicular (SC) and acromioclavicular (AC) joints (Inman and Saunders, 1946), causing the scapula to both rotate and translate over the thorax. Recent three dimensional (3D) kinematic techniques have confirmed these clinical observations and have demonstrated the importance of scapular motion for full, functional mobility of the arm (Pronk et al., 1993; van der Helm and Pronk, 1995; Ludewig et al., 1996; McClure et al., 2001; Ludewig et al., 2004; Magermans et al., 2005; Ludewig et al., 2009; Roren et al., 2012). In addition, studies have demonstrated that scapular kinematics are altered in most pathological conditions that affect the shoulder (rotator cuff pathologies, shoulder instability and shoulder stiffness); see review in Ludewig and Reynolds, (2009).

The description of scapular motion relative to the thorax remains, however, a scientific and clinical challenge. The International Society of Biomechanics (ISB) has proposed a protocol based on Euler angle formalism for the geometrical description of the kinematic chain (van der Helm, 1997, Wu et al., 2005). Scapulothoracic motion is described by two distinct sequences of three Euler angles: one sequence for the rotations of the clavicle relative to the thorax (proretraction, elevation-depression, and axial rotation at the SC joint), and the other for the rotations of the scapula relative to the clavicle (pro-retraction, medial-lateral rotation and anterior-posterior tilt at the AC joint). Scapular motion can also be described relative to the trunk (thus neglecting clavicular motion) using the same sequence. There are, however three problems related to the ISB protocol.

First, the clinical usability of the ISB model is limited by the fact it is based on the use of data obtained either by invasive recording methods or by external, but imprecise and incomplete methods. The ISB protocol is based on the measurement of the 3D position of two bony landmarks which define the clavicle (most ventral point on the SC joint and most dorsal point on the AC joint) and three bony landmarks which define the scapula (angle of the acromion, root of the scapular spine and inferior angle of the scapula). The protocol was validated using sensors inserted directly into the bones (Karduna et al., 2001, Mc Clure 2001, Ludewig et al.,2009). Using this technique, Ludewig et al. (2009) showed that substantial rotations occur in all the DoF of the SC and AC joints. However, it is not possible to insert sensors into the bones in clinical practice. Biplane fluoroscopy (Giphart et al., 2013) is another highly reliable technique but cannot be used in clinical practice either. 
Motion capture of the scapula using external sensors fixed over bony landmarks is subject to artefacts caused by skin sliding and muscle bulging (Van Andel et al., 2009). A well accepted method for the capture of scapular motion consists of using one 6 DoF sensor fixed over the acromion process to define the position and orientation (i.e. $6 \mathrm{DoF}$ ) of an acromial reference frame ("acromial method") (Karduna at al., 2001). Bony landmarks of the scapula are then digitized and computed in the acromial reference frame before being projected in the global 3D space (Meskers et al., 1998). This method allows real continuous dynamic tracking of scapular motion during natural movements (Karduna et al., 2001; Meskers et al., 2007). Several types of 6 DoF sensors have been used in studies: electromagnetic sensors (Meskers et al., 1998; Fayad et al., 2006), inertial devices (Parel et al., 2012) and optical marker clusters (van Andel et al., 2009; Lempereur et al., 2010; Senk and Cheze, 2010). The level of error associated with acromial methods is acceptable for ranges of arm elevation below $120^{\circ}$ (Karduna et al., 2001; Ludewig et al., 2009). These methods can be used to assess 3D shoulder kinematics in a variety of conditions in healthy subjects (Dayanidhi et al., 2005; Fayad et al., 2006; Yano et al., 2010) and in patients with different shoulder pathologies (Ludewig and Cook, 2000 ; Lin et al ., 2006; Rundquist, 2007; Amasay and Karduna, 2009). The analysis of clavicular function is usually limited to the 2D static measurement of protraction and elevation (2 DOF) using imaging or goniometric methods (Conway, 1961; Inman et al., 1996; Hallaceli and Gunal, 2002). Motion capture of the clavicle with external markers is very new. Two studies in the literature have used clavicle motion capture techniques. One technical study proposed the 2D dynamic measurement of clavicular rotations using the acromial method with the digitization of two additional landmarks (AC and SC) (Helgadottir et al., 2010). The other study presented SC and AC joint rotations in healthy subjects (Teece et al., 2008). The quantification of axial rotations of the clavicle is particularly challenging due to skin artefacts and the crank-shape of the clavicle (Ludewig et al., 2004, Helgadottir et al., 2011).

In the present study, we propose to extend the acromial method in order to capture, in addition to scapular rotations, the linear translations (displacements) of the centre of the scapula relative to the thorax. This technique yields $6 \mathrm{DoF}$, thus providing a direct and complete measurement of the kinematics of the shoulder complex, including the clavicle.

The second problem with the ISB protocol relates to the inconsistent use of clinical and biomechanical terminology. In anatomical and clinical descriptions, scapula motion is reported to consist of three rotations and two translations (Peat, 1986; Culham and Peat, 1993; Sahrmann, 2001; Tytherleigh-Strong, 2009). The rotation of the scapula in its main plane is often called upward/downward rotation (sometimes lateral and medial rotation), the other rotations involve winging around the vertical axis: internal and external rotation (sometimes named protraction/retraction) and tilting around a horizontal axis (anterior/posterior tilt or tilting or, less frequently, tipping). The translations are reported as elevation/depression (sometimes 
named upward/downward translation) and abduction-adduction (toward/away from the vertebral spine). According to some authors, protraction is not a pure rotation but a combination of rotation and translation involving both scapular and clavicular motion. It is defined as " $a$ forward movement of the scapula around the thoracic wall [which] combines linear translation away from the vertebral column, rotation of the scapula around the end of the clavicle (winging) and anterior movement of the lateral end of the clavicle" (Culham and Peat, 1993) see also (Solem-Bertoft et al., 1993). Clinical observations thus incite the inclusion of translations in the kinematic description of global scapula motion.

The last problem of the ISB protocol relates to the dimensions (i.e. the minimum number of DoF) needed to describe scapular motion relative to the trunk. Theoretically, the motion of a solid in space is defined by 6 parameters: 3 for position and 3 for orientation. The ISB model using 6 DoF within an open kinematic chain thus provides a sufficient description of scapula motion relative to the trunk (Bao and Willems, 1999). However, the shoulder complex is a closed chain due to the contact between the scapula and the thorax. The anatomy of the bony and musculo-ligamentous structures is likely to constrain the motion of the scapula in space and to create couplings, limiting the effective dimensions of scapulothoracic motion. Several mechanical models of these constraints have been proposed. However, there is no agreement on the minimum number of independent DoF needed for the description of scapulothoracic motion; 4 according to van der Helm (1994) and Seth et al. (2010, 2012); 5 according to Bao and Willems (1999) and 6 according to Lenarcic and Stanisic (2003). To our knowledge, this question has not been investigated experimentally using kinematic methods.

The present study had a double purpose. The first aim was to completely describe and quantify the motion of the scapula relative to the trunk, including both scapular rotations and translations (linear displacements of the centre of the scapula (CS)).

The second aim was to specify the "functional dimension" of scapular motion during a large variety of motor tasks in healthy subjects. For this, movements involving analytical arm elevation in the sagittal and frontal planes, simulations of activities of daily living (hair combing (HC) and back washing (BW) and the maximum voluntary scapula-thoracic movement in different directions (forward and backward shoulder rolling: F-Roll and B-Roll) were recorded and analysed. The hypothesis was that 6 DoF might not be necessary for the description of scapulothoracic motion, due to functional coupling between rotations and translations. 


\section{METHOD}

\section{Population}

A convenience sample of eight healthy volunteers (age $31.14 \pm 9.30$ years, range 24-50; height $1.72 \pm 0.82 \mathrm{~m}$, range 1.62-1.87; weight $66.71 \pm 9.11 \mathrm{~kg}$, range 54-82; BMI $22.55 \pm 2.31$ $\mathrm{kg} / \mathrm{m}^{2}$, range 19.13-25.91) with no prior history of upper extremity disorders took part in this study. All the subjects were right-handed, healthy and active adults; they were all working in the rehabilitation unit. The study protocol was approved by the local Institutional Review Board and all subjects provided informed consent.

\section{Instrumentation}

Real-time 3-D position and orientation of the thorax, scapula and humerus were tracked (30$\mathrm{Hz}$ sampling rate) using the four sensors of the Polhemus Fastrak electromagnetic device. The reported root mean square (RMS) accuracy of this system is $0.3-0.8 \mathrm{~mm}$ for position and $0.15^{\circ}$ for orientation when used within a $0.76 \mathrm{~m}$ source to sensor separation (SPACE FASTRAK User's Manuel, Revision F. Colchester, VT; Polhemus Inc.; 1993). The transmitter, which provided the general frame of reference, was fitted on a rigid plastic frame to an adjustable camera tripod placed in front of the subject. The reference frame was positioned approximately at the level of the navel, so that the sensors were within a range of $0.3 \mathrm{~m}$ to $0.7 \mathrm{~m}$ from the transmitter.

\section{Experimental procedure}

Kinematics. The thoracic and scapular sensors were fixed on the patient's skin over the sternum and the flat surface of the superior acromion process respectively. The humeral sensor was strapped to the arm with Velcro, just below the insertion of the deltoid. The fourth sensor, mounted on a pointer, was used to manually digitize the bony landmarks palpated on the thorax (xiphoid process, suprasternal notch, and spinal processes of C7 and T8), scapula (acromial angle, root of the spine and inferior angle) and humerus (medial and lateral epicondyle). The local coordinates of the bony landmarks were computed in the reference frame of the corresponding sensor (Mc Clure 2001; Fayad et al., 2006). The centre of the gleno-humeral joint was computed by a regression method (Biryukova et al., 2000). Joint rotation matrices between the reference frames based on the bony landmarks of the rigid bodies were then computed and the rotations expressed using Euler angle sequences (van der Helm, 1997). Scapular rotations were: internal/external rotation ${ }^{1}$ around the main thoracic axis, then medial/lateral rotation

\footnotetext{
${ }^{1}$ According to Ludewig (2009) instead of «pro/retraction ».
} 
around an axis perpendicular to the scapular plane, followed by anterior/posterior tilt (Fig. 1). The 3D coordinates of the centre of the scapula (CS) (i.e. the barycentre of the triangle representing the scapula) were calculated in the reference frame of the thorax. Thus, the displacement of CS was expressed by projections in three directions (forward, lateral and upward) relative to the centre of the thorax (barycentre of the four points: xiphoid process, suprasternal notch, and spinal processes of C7 and T8) (Hanneton et al., 2011). Arm elevation relative to the thorax was also computed as the angle between the main axis of the humerus and that of the trunk irrespective of the plane of elevation. Left side data were projected to the right side. To that purpose, the symmetrical 3D positions of all the anatomical landmarks with respect to the sagittal plane were calculated before further computations.

Recording procedure. Skin preparation and fixation of the markers on the skin surface (which is the longest phase of the whole procedure) were carried out with subjects seated to avoid fatigue.

During the digitization process, the subjects were asked to adopt a reference position: standing upright with both arms hanging beside the body. Then, kinematic data were recorded at $30 \mathrm{~Hz}$ while the following movements were carried out in the same order: for forward rolling (FRoll), the subjects were instructed to perform the largest possible movement of the scapula in the forward, upward and backward directions, keeping their arm by their sides. For backward rolling (B-Roll) the instruction was the same for the reverse succession of backward upward and forward directions (Sheikhzadeh et al., 2008). The subjects were then instructed to perform maximal active analytic arm elevation: first in the sagittal plane (Flexion) then in the frontal plane (Abduction). Finally, they were instructed to simulate two activities of daily living: hair combing (HC) and back washing (BS) (Roren et al., 2012). Before each recording, each movement was described to the subject who then performed it twice for a warm-up and to ensure he/she carried out the movement as instructed: avoid flexing the elbow or moving the trunk, complete the movement in about $10 \mathrm{~s}$ and remain in a fixed plane of motion for analytical arm elevation. During the recording, the observer gave permanent verbal support so that the subject achieved the movement within the recording time and remained in the correct plane of motion. Incorrect trials were repeated in order to obtain two analysable trials. The recording of the tasks lasted less than 10 minutes for each side. The whole procedure was carried out for both shoulders, starting with the right side, and lasted a maximum of 60 minutes.

Data analysis. Kinematic data (arm elevation, scapulothoracic rotations and CS translations) were analyzed using custom made interactive software (Labview 8.2). The values of arm elevation were used to compute the beginning ( $\mathrm{t} 0$ ) and end (tf) of each movement with a threshold equal to 3 times the standard deviation of the resting value (measured during the first ten recording samples). Two time points were determined for Flexion, Abduction, BW and HC. The first (t1) corresponded to the initial part of the movement computed at an arm elevation of 
$90^{\circ}$ (Flexion, Abduction and $\mathrm{HC}$ ) or at the first maximum (BW). The second ( $\mathrm{t} 2$ ) corresponded to the final posture characteristic of the task. Three time points (t1-3) were determined for FRoll and B-Roll, relating to the three phases of the roll.

For the data analysis, the mean of the values obtained for the two successive repetitions of each task was used. Then, the means within the group of subjects were computed for each side and each task. Statistical analysis (ANOVA and Student's t-test) was carried out using Statview. The significance level was fixed at alpha $=0.05$.

The arm elevation angle and the 6 scapulothoracic parameters were computed at each of the time points ( $\mathrm{t} 0$ to $\mathrm{t} 2$ or $\mathrm{t} 3$ ). Changes in values were then calculated relative to the initial values at $\mathrm{t} 0$. The absolute amplitude of the 3D translation of the CS was also calculated.

In addition, the kinematic data corresponding to each movement were time-normalized to 100 time intervals between $\mathrm{t} 0$ and $\mathrm{tf}$ in order to remove spontaneous variations in movement duration. Time normalized signals were averaged within subjects and sides. Principal Component Analysis (PCA) was used to investigate the dimensionality of the data. The mathematical procedure of the PCA transforms a number of possibly correlated variables into a smaller number of uncorrelated variables called principal components (PC, PCs in the plural), ranked according to the amount of variance explained by each. PCA was performed with varimax transformation (Statview software) on the normalized time course of the 6 scapulothoracic parameters cumulating all the tasks (i.e. on a matrix with 6 columns and $100 \times 6$ lines). This analysis yields the amount of variance explained by each PC and the correlation between PCs and DoF. 


\section{$\underline{\text { RESULTS }}$}

The 6 DoF describing the initial posture of the scapula relative to the thorax were analyzed using a two factors ANOVA (side and task) with participants as repeated measures. For scapula internal rotation, there was a significant effect of task (F72, 5=5.2, $\mathrm{p}<0.001$, but not side $(F 72,1=0.8)$. Post-Hoc analysis showed that the scapula was less internally rotated at the beginning of the B-Roll than for the other tasks $\left(-4.6^{\circ} \pm 0.5^{\circ}\right.$, non significant, ns). For the other DoF, there was no effect of task $(\mathrm{F} 72,1<1.6, \mathrm{~ns})$ or side $(\mathrm{F} 72,1<0.9, \mathrm{~ns})$. In addition, there were no significant differences between sides, for movement at $\mathrm{t} 1$ and $\mathrm{t} 2$, whatever the task (Student's $t$ test). Thus data from the two sides were averaged and this average was used for the rest of the analysis.

The time course of humerothoracic elevation and the selected time points are shown in Fig. 2. For the Flexion, Abduction and HC tasks there was a raising and a lowering of the humerus relative to the thorax. For the BW task, arm elevation increased then decreased to complete the task, then followed the reverse pattern for the return movement. The B-Roll and F-Roll tasks both followed a tri-phasic pattern, as expected from the instructions given. The time points ( $\mathrm{t} 1$ to t3) were placed in the middle of the plateau (or at the local maxima, if any) for each phase.

An example of scapula kinematics during the different tasks is shown for a representative subject in Fig. 3. The normalised time courses of humerothoracic elevation (Fig. 2) and scapula rotation angles during the different tasks are illustrated in Fig. 4-6. The amount of rotation between the initial posture and $\mathrm{t} 1-\mathrm{t} 3$ are summarized in Tables 1 and 2 .

During Abduction (Fig. 4, black lines), there was a large increase in scapular lateral rotation $\left(29.64 \pm 1.60^{\circ}\right)$, accompanied by a small amount of external rotation $\left(-4.06 \pm 1.07^{\circ}\right)$ and posterior tilting $\left(9.57 \pm 0.79^{\circ}\right)$. The small translation of the CS $(10.0 \pm 1.1 \mathrm{~mm}$ in $3 \mathrm{D})$ was mainly medial $(-4.2 \pm 1.2 \mathrm{~mm}$ ). During Flexion (Fig. 4, grey lines), there was also a large amount of lateral rotation $\left(27.06 \pm 1.24^{\circ}\right)$ accompanied by internal rotation $\left(16.86 \pm 0.78^{\circ}\right)$ and posterior tilting $\left(5.02 \pm 1.36^{\circ}\right)$. The CS moved laterally and forward $(24.5 \pm 1.8 \mathrm{~mm}, 16.1 \pm$ $2.1 \mathrm{~mm}$, respectively), corresponding to a total 3D amplitude of $31.8 \pm 1.2 \mathrm{~mm}$.

The results obtained for simulated activities of daily living are illustrated in Fig. 5. During simulated hair combing (Fig. 5, HC, grey lines), the movement consisted mainly of a large amount of lateral rotation $\left(32.03 \pm 1.05^{\circ}\right)$ and posterior tilt $\left(6.36 \pm 0.81^{\circ}\right)$. The CS moved mainly laterally $(12.6 \pm 1.8 \mathrm{~mm})$. During the initial elevation, the scapula rotated medially ($\left.6.11 \pm 0.47^{\circ}\right)$ and anterior tilt increased $\left(-8.06 \pm 0.74^{\circ}\right)$. The translation of CS was medial $(-8.7$ $\pm 1.2 \mathrm{~mm})$ and mainly upward $(27.6 \pm 1.9 \mathrm{~mm})$.

Shoulder rolling involved maximal voluntary movements of the scapula in three successive phases (Fig. 6 and Table 2). During F-Roll (Fig.6, grey lines), the first phase was characterized 
at $\mathrm{t} 1$ by scapular internal rotation $\left(22.36 \pm 1.68^{\circ}\right)$ and scapular lateral rotation $\left(21.34 \pm 1.49^{\circ}\right)$, and was accompanied by a large translation of the CS in all directions $(77.8+6.8 \mathrm{~mm}$ in 3D). The second phase was characterized at $\mathrm{t} 2$ by a further increase in lateral rotation $\left(39.67 \pm 1.76^{\circ}\right)$ accompanied by anterior tilting $\left(-25.62+1.92^{\circ}\right)$ and upward CS translation $(96.8 \pm 6.8 \mathrm{~mm})$ while internal rotation decreased $\left(21.10 \pm 2.72^{\circ}\right)$. The maximum scapular translation occurred at this time $(108.9+7.9 \mathrm{~mm}$ in 3D). The last phase was characterized at $\mathrm{t} 3$ by a large scapular external rotation $\left(-43.51 \pm 1.18^{\circ}\right)$, a decrease in lateral rotation $\left(15.59 \pm 1.06^{\circ}\right)$ and a posterior tilt $\left(3.73 \pm 0.67^{\circ}\right)$. The CS moved medially $(-84.4 \pm 3.3 \mathrm{~mm})$ and backwards $(-33.1 \pm 2.3 \mathrm{~mm})$ (92.6 $\pm 3.3 \mathrm{~mm}$ in 3D). During B-Roll (Fig.6, black lines), scapular kinematics were similar, in the reverse order.

Coupling between the 6 DoF of scapulothoracic motion was investigated during the different movements using PCA. PCA extracts the common factors of the time course of the 6 DoF during all the tasks. Three PCs were identified, explaining respectively $47.4 \%$ (PC1), $31.5 \%$ (PC2) and $18.7 \%$ (PC3) of the variance for a total of $97.6 \%$. PC1 was correlated with internal rotation $(r=0.866)$, lateral and forward CS translation ( $r=0.951$ and $r=0.687$, respectively) but not the other DoF $(r<0.042)$. PC2 was mainly correlated with upward translation $(r=0.970)$ and anterior tilt ( $\mathrm{r}=-0.889)$ and more weakly with forward and medial translation, ( $\mathrm{r}=0.456$ and $\mathrm{r}=-$ 0.501 respectively). PC3 was mainly correlated with medial/lateral rotation $(\mathrm{r}=0.984)$. 


\section{DISCUSSION}

The current study showed, in a variety of tasks, that scapulothoracic motion involves both $3 \mathrm{D}$ rotations of the scapula and significant 3D translations. The direction of the 3D linear translation of the CS was specific to each task and functionally consistent.

The patterns of 3D scapular rotation recorded in this study corroborate the findings of previous 3D studies which also evaluated scapular kinematics during planar arm elevation and simulated tasks such as hair-combing and back-washing (Triffitt, 1998; Magermans et al., 2005; Fayad et al., 2006; Fayad et al., 2008; Rundquist et al., 2009; Roren et al., 2012). Similarly to the results of a previous study (Sheikhzadeh et al., 2008), the present study showed that shoulder rolling provoked much larger scapular internal/external rotation and anterior/posterior tilt than other arm movements. In addition, the present study provides original data regarding 3D translations of the CS during analytical arm movements and ADL. Translations of the CS remained below $30 \mathrm{~mm}$ when accompanying arm movements. This is in accordance with a previous study that assessed 3D scapular rotations and 2D translations using an electromechanical digitizer at three different degrees of arm elevation (Wang et al., 1999). In contrast, during shoulder rolling, when the instruction was to voluntarily perform the largest possible scapular movements, there were large translations of the CS which could reach $~ 100$ $\mathrm{mm}$.

The description of scapula motion used in the present study (three rotations and the translation of its barycentre) is geometrically correct and does not involve any hypothesis regarding the position of the instantaneous axis of rotation, which is known to vary during arm movements (Culham and Peat, 1993), or any possible translation along the instantaneous axis of rotation (Woltring et al., 1985).

The current study used an acromial method with electromagnetic sensors. Karduna et al. (2001) found that errors due to skin artefacts were relatively small for arm elevation up to $120^{\circ}$. Moreover, reliability studies of scapular motion during analytical arm elevation have shown fair to excellent repeatability in healthy subjects (McQuade and Smidt, 1998; Tsai et al., 2003; Fayad et al., 2006; Roren et al., 2013). Most of the variables analyzed in the present study were recorded below $120^{\circ}$ of elevation, except for maximum elevation during Flexion and Abduction (time t2). However, the good correlations obtained with the PCA showed that the scapular kinematics obeyed similar coupling laws whatever the task. The greatest scapular translations and rotations occurred during the shoulder rolling tasks, as specified by the instruction. It must be emphasised that these tasks were performed well below the level of arm elevation which might create skin artefacts. 
The current study confirms the empirical descriptions of well coordinated scapular rotations and translations (Culham and Peat, 1993) and quantifies the remarkable range of motion and flexibility of the shoulder complex. The combined movements of the scapula result from the mobility of the clavicle which is linked to the scapula via the SC and AC joints (Inman and Saunders, 1946; Ludewig et al., 2009; Teece,et al., 2008). However, the relationship between motion of the clavicle (at the AC and SC joints) and motion of the scapula relative to the thorax is not simple, particularly because of the crank-shape of the clavicle. Axial rotation around the SC joint may cause significant displacement of the position of the AC joint and thus of the scapula as a whole (rotation and translation of CS). For the first time, the present method affords a compact expression of $6 \mathrm{D}$ scapulothoracic kinematics: the measurement of the linear translation of the CS, in addition to 3D scapular rotations. It also provides an indirect, non invasive and global assessment of clavicular motion. This computation can be easily implemented in any biomechanical method which provides the 3D position of the scapular landmarks according to the ISB protocol.

The fact that CS translations occurred during all the tasks suggests that clavicle motion occurs extremely frequently in daily life, possibly leading to local constraints at the AC and SC joints. The prevalence of $\mathrm{AC}$ and $\mathrm{SC}$ arthritis in asymptomatic subjects is high (Kier et al., 1996; Needell et al., 1996; Stein et al., 2001). This prevalence seems to be explained by the high and repetitive loads which occur during activities of daily living, combined with anatomical factors such as the narrowness and incongruence of the articular surfaces (Grant, 1965; Corteen and Teitge, 2005; Docimo et al., 2008; Colegate-Stone et al., 2010). The kinematic protocol proposed here provides an indirect assessment of 3D clavicular motion which could be useful to improve understanding of loading and pathologies of the AC and SC joints.

The PCA showed that the functional dimension of shoulder girdle movements may be limited to 3 principal components. The first factor, $\mathrm{PC} 1$ combined internal/external rotation and forward/backward and medial/lateral translation, consistent with the classical anatomical and clinical definitions of "protraction-retraction". PC2 combined anterior/posterior tilting with upward/downward translation (clinically termed shoulder shrugging). PC3 represented medial/lateral rotation. This therefore suggests that internal/external rotation and medial/lateral translation are strongly coupled and that only $3 \mathrm{DoF}$ are needed to describe scapular movement in healthy subjects.

The coupling evidenced by the PCA could either be the result of anatomical factors constraining scapular motion around the thoracic wall and/or the synergic organisation of movement by the central nervous system (Latash, 2008); two hypotheses which are not incompatible. Indeed, it is known that both central neural networks and local biomechanical constraints shape the control of movement. This is the case, for example for synergies of the 
hand and fingers (Santello et al., 2013) and control of the hand/arm trajectory (Schaal and Sternad, 2001).

\section{Limitations:}

The small size of the convenience sample included must be considered in the interpretation of the results. Further studies are needed in a larger group of healthy subjects, taking into account their size or other anthropomorphic factors. The lack of control of movement velocity during the tasks could be a limitation; however, the humerothoracic and scapular recordings were time normalized. Moreover, it has been shown that the velocity of arm elevation does not modify 3D scapular kinematics (Fayad et al., 2006). The order of the tasks and sides (left-right) were not randomized, however, the recording procedure was not likely to generate fatigue in these healthy subjects and no significant differences for scapular rotations or translations were found between sides.

\section{CONCLUSION}

The functional decomposition of DoF evidenced by the PCA provides an innovative model of scapular motion. It showed that scapular motion involves simultaneous translations and rotations, constrained by the curved surface of the thorax and likely also by muscles and ligaments: the first two PCs summarized motion on this surface and the third PC related to rotation tangential to this surface. This mechanical hypothesis is consistent with the model proposed by Seth et al. $(2010 ; 2012)$ who used 4 DoF to simulate scapular movement over the thorax, represented by an ellipsoid, and fits the data by Ludewig et al. (2009). The present study suggests that 3 DoF may be sufficient in order to fully describe scapular motion in healthy subjects. Further studies with precise mechanical models are needed to better understand the contribution of the different elements of the shoulder complex to the kinematics of the arm.

The fine coordination between clavicular and scapular motion sub serving this coupling might be disrupted in pathological conditions. In the future, the analysis of CS translation should be used as a complement to acromial methods for the evaluation of shoulder kinematics in patients with different pathologies of the shoulder complex (Ludewig and Cook, 2000; Vermeulen et al., 2002; Lin et al., 2006; Fayad et al., 2008). 


\section{REFERENCES}

Amasay T, Karduna AR. Scapular kinematics in constrained and functional upper extremity movements. Journal of Orthopaedic \& Sports Physical Therapy 2009; 39(8): 618-27.

Bao H, Willems PY. On the kinematic modelling and the parameter estimation of the human shoulder. Journal of Biomechanics 1999; 32(9): 943-50.

Biryukova EV, Roby-Brami A, Frolov AA, Mokhtari M. Kinematics of human arm reconstructed from spatial tracking system recordings. Journal of Biomechanics 2000; 33(8): 985-95.

Codman M. The Shoulder. Boston: Thomas Todd Co; 1934. p. 1-513.

Colegate-Stone T, Allom R, Singh R, Elias DA, Standring S, Sinha J. Classification of the morphology of the acromioclavicular joint using cadaveric and radiological analysis. The Journal of Bone and Joint Surgery Br. 2010; 92(5): 743-6.

Conway AM. Movements at the sternoclavicular and acromioclavicular joints. Physical Therapy Reviews 1961; 41: 421-32.

Corteen DP, Teitge RA. Stabilization of the clavicle after distal resection: a biomechanical study. American Journal of Sports Medicine 2005; 33(1): 61-7.

Culham E, Peat M. Functional anatomy of the shoulder complex. The Journal of Orthopaedic and Sports Physical Therapy. 1993; 18(1): 342-50.

Dayanidhi S, Orlin M, Kozin S, Duff S, Karduna A. Scapular kinematics during humeral elevation in adults and children. Clinical Biomechanics (Bristol, Avon) 2005; 20(6): 600-6.

Docimo S Jr, Kornitsky D, Futterman B, Elkowitz DE. Surgical treatment for acromioclavicular joint osteoarthritis: patient selection, surgical options, complications, and outcome. Current Reviews in Musculoskeletal Medicine 2008; 1(2): 154-60.

Fayad F, Hoffmann G, Hanneton S, Yazbeck C, Lefevre-Colau MM, Poiraudeau S, Revel M, Roby-Brami A. 3-D scapular kinematics during arm elevation: effect of motion velocity. Clinical Biomechanics (Bristol, Avon) 2006; 21(9): 932-41.

Fayad F, Roby-Brami A, Yazbeck C, Hanneton S, Lefevre-Colau MM, Gautheron V, Poiraudeau S, Revel M. Three-dimensional scapular kinematics and scapulohumeral rhythm in patients with glenohumeral osteoarthritis or frozen shoulder. Journal of Biomechanics 2008; 41(2): 326-32.

Giphart J, Brunkhorst J, Horn N, Shelburne K, Torry M, Millett P. Effect of plane of arm elevation on glenohumeral kinematics: a normative biplane fluoroscopy study. The Journal of Bone and Joint Surgery Am. 2013; 95(3): 238-45.

Grant JCB. Method of anatomy, 7th ed. Baltimore: Williams and Wilkins, 1965. p. 1-777. 
Hallaceli H, Gunal I. Normal range of scapular elevation and depression in healthy subjects. Archives of Orthopaedic and Trauma Surgery 2002; 122(2): 99-101.

Hanneton S, Dedobbeler S, Hoellinger T, Roby-Brami A. Direct kinematic modeling of the upper limb during trunk-assisted reaching. Journal of Applied Biomechanics 2011; 27(3): 2727.

Helgadottir H, Kristjansson E, Mottram S, Karduna AR, Jonsson H Jr. Altered scapular orientation during arm elevation in patients with insidious onset neck pain and whiplashassociated disorder. The Journal of Orthopaedic and Sports Physical Therapy 2010; 40(12): 784-91.

Helgadottir H, Kristjansson E, Mottram S, Karduna A, Jonsson H, Jr. Altered alignment of the shoulder girdle and cervical spine in patients with insidious onset neck pain and whiplashassociated disorder. Journal of Applied Biomechanics 2011; 27(3): 181-91.

Inman V, Saunders J. Observations on the function of the clavicle. California Medicine 1946; 65(4): 158-66.

Inman VT, Saunders JB, Abbott LC. Observations of the function of the shoulder joint. 1944. Clinical Orthopaedics and Related Research. 1996; 330: 3-12.

Karduna AR, McClure PW, Michener LA, Sennett B. Dynamic measurements of threedimensional scapular kinematics: a validation study. Journal of Biomechanical Engineering 2001; 123(2): 184-90.

Kier R, Wain SL, Apple J, Martinez S. Osteoarthritis of the sternoclavicular joint. Radiographic features and pathologic correlation. Investigative Radiology 1986; 21(3): 227-33.

Latash ML. Synergy. New York: Oxford University Press; 2008. p. 1-412.

Lempereur M, Brochard S, Burdin V, Remy-Neris O. Difference between palpation and optoelectronics recording of scapular motion. Computer Methods in Biomechanics and Biomedical Engineering 2010; 13(1): 49-57.

Lenarcic J, Stanisic M. A Humanoid Shoulder Complex and the Humeral Pointing Kinematics, IEEE Transactions on Robotics and Automation. 2003; 19(3): 499-506.

Lin JJ, Hanten WP, Olson SL, Roddey TS, Soto-Quijano DA, Lim HK, Sherwood AM. Shoulder dysfunction assessment: self-report and impaired scapular movements. Physical Therapy 2006; 86(8): 1065-74.

Ludewig PM, Behrens SA, Meyer SM, Spoden SM, Wilson LA. Three-dimensional clavicular motion during arm elevation: reliability and descriptive data. Journal of Orthopaedic \& Sports Physical Therapy 2004; 34(3): 140-9.

Ludewig PM, Cook TM. Alterations in shoulder kinematics and associated muscle activity in people with symptoms of shoulder impingement. Physical Therapy 2000; 80(3): 276-91. 
Ludewig PM, Cook TM, Nawoczenski DA. Three-dimensional scapular orientation and muscle activity at selected positions of humeral elevation. The Journal of Orthopaedic and Sports Physical Therapy 1996; 24(2): 57-65.

Ludewig P, Phadke V, Braman J, Hassett D, Cieminski C, Laprade R. Motion of the shoulder complex during multiplanar humeral elevation. The Journal of Bone and Joint Surgery Am. 2009; 91 (2): 378-89.

Ludewig PM, Reynolds JF. The association of scapular kinematics and glenohumeral joint pathologies. Journal of Orthopaedic \& Sports Physical Therapy 2009; 39(2): 90-104.

Magermans DJ, Chadwick EK, Veeger HE, van der Helm FC. Requirements for upper extremity motions during activities of daily living. Clinical Biomechanics (Bristol, Avon) 2005; 20(6): 591-99.

McClure PW, Michener LA, Sennett BJ, Karduna AR. Direct 3-dimensional measurement of scapular kinematics during dynamic movements in vivo. Journal of Shoulder and Elbow Surgery 2001; 10(3): 269-77.

McQuade KJ, Smidt GL. Dynamic scapulohumeral rhythm: the effects of external resistance during elevation of the arm in the scapular plane. Journal of Orthopaedic and Sports Physical Therapy 1998; 27(2): 125-33.

Meskers CG, van de Sande MA, de Groot JH. Comparison between tripod and skin-fixed recording of scapular motion. Journal of Biomechanics 2007; 40(4): 941-46.

Meskers CG, Vermeulen HM, de Groot JH, van Der Helm FC, Rozing PM. 3D shoulder position measurements using a six-degree-of-freedom electromagnetic tracking device. Clinical Biomechanics (Bristol, Avon) 1998; 13(4-5): 280-92.

Needell SD, Zlatkin MB, Sher JS, Murphy BJ, Uribe JW. MR imaging of the rotator cuff: peri tendinous and bone abnormalities in an asymptomatic population. American Journal of Roentgenology 1996; 166(4): 863-7.

Parel I, Cutti AG, Fiumana G, Porcellini G, Verni G, Accardo AP. Ambulatory measurement of the scapulohumeral rhythm: intra- and inter-operator agreement of a protocol based on inertial and magnetic sensors. Gait \& Posture. 2012; 35(4): 636-40.

Peat M. Functional anatomy of the shoulder complex Physical Therapy 1986; 66(12): 185565 .

Pronk GM, van der Helm F, Rozendaal L. Interaction between the joints in the shoulder mechanism: the function of the costoclavicular, conoid and trapezoid ligaments. Proceedings of the Institution of Mechanical Engineers Part H: Journal of engineering in medicine; 1993; 207(4): 219-29.

Roren A, Fayad F, Roby-Brami A, Revel M, Fermanian J, Poiraudeau S, Robertson J, Lefevre-Colau MM. Precision of 3D scapular kinematic measurements for analytic arm movements and activities of daily living. Manual Therapy 2013; 18(6): 473-80. 
Roren A, Lefevre-Colau MM, Roby-Brami A, Revel M, Fermanian J, Gautheron V, Poiraudeau S, Fayad F. Modified 3D scapular kinematic patterns for activities of daily living in painful shoulders with restricted mobility: a comparison with contralateral unaffected shoulders. Journal of Biomechanics 2012; 45(7): 1305-11.

Rundquist, PJ. Alterations in scapular Kinematics in subjects with idiopathic loss of shoulder range of motion. Journal of Orthopaedic and Sports Physical Therapy 2007; 37(1): 19-25.

Rundquist PJ, Obrecht C, Woodruff L. Three-dimensional shoulder kinematics to complete activities of daily living. American Journal of Physical Medicine \& Rehabilitation 2009; 88(8): 623-29.

Sahrmann S. Diagnosis and Treatment of Movement Impairment Syndromes by Shirley Saint Louis, USA: Mosby; 2001.

Santello M, Baud-Bovy G, Jörntell H. Neural bases of hand synergies. Frontiers in Computational Neuroscience 2013; 7(23): 1-15.

Schaal S, Sternad D. Origins and violations of the $2 / 3$ power law in rhythmic threedimensional arm movements. Experimental Brain Research 2001; 136(1): 60-72.

Senk M, Cheze L. A new method for motion capture of the scapula using an optoelectronic tracking device: a feasibility study. Computer Methods in Biomechanics and Biomedical Engineering 2010; 13(3): 397-401.

Seth A, Matias R, Veloso A, Delp S. A scapulothoracic joint model for fast and accurate simulations of upper-extremity motion. ASB 2012, American society of Biomechanics, Gainesville, Florida, 2012. http://www.asbweb.org/conferences/2012/abstracts/12.pdf

Seth A, Sherman M, Eastman P, Delp S. Minimal formulation of joint motion for biomechanisms. Nonlinear Dynamics 2010; 62(1): 291-303.

Sheikhzadeh A, Yoon J, Pinto VJ, Kwon YW. Three-dimensional motion of the scapula and shoulder during activities of daily living. Journal of Shoulder and Elbow Surgery 2008; 17(6): 936-42.

Solem-Bertoft E, Thuomas KA, Westerberg CE. The influence of scapular retraction and protraction on the width of the subacromial space. An MRI study. 1993; 296: 99-103.

SPACE FASTRAK User's Manuel, Revision F. Colchester, VT; Polhemus Inc.; 1993

Stein BE, Wiater JM, Pfaff HC, Bigliani LU, Levine WN. Detection of acriomioclavicular joint pathology in asymptomatic shoulders with magnetic resonance imaging. Journal of Shoulder and Elbow Surgery 2001; 10(3): 204-8.

Teece RM, Lunden JB, Lloyd AS, Kaiser AP, Cieminski CJ, Ludewig PM. Threedimensional acromioclavicular joint motions during elevation of the arm. Journal of Orthopaedic and Sports Physical Therapy 2008; 38(4): 181-90.

Triffitt $\mathrm{P}$. The relationship between motion of the shoulder and the stated ability to perform activities of daily living. The Journal of Bone and Joint Surgery Am. 1998; 80(1): 41-6. 
Tsai NT, McClure PW, Karduna AR. Effects of muscle fatigue on 3-dimensional scapular kinematics. Archives of Physical Medicine and Rehabilitation 2003; 84(7): 1000-5.

Tytherleigh-Strong G. Pectoral girdle, shoulder region and axilla. In: Standring S, Borley NR, Collins P et al., editors. Gray's Anatomy: The Anatomical Basis of Clinical Practice. London: Churchill Livingstone Elsevier, 2008. p.803.

van Andel C, van Hutten K, Eversdijk M, Veeger D, Harlaar J. Recording scapular motion using an acromion marker cluster. Gait \& Posture. 2009; 29(1): 123-8.

van der Helm FC. A finite element musculoskeletal model of the shoulder mechanism. Journal of Biomechanics 1994; 27(5): 551-69.

van der Helm FC, Pronk GM. Three-dimensional recording and description of motions of the shoulder mechanism. The Journal of Biomechanical Engineering 1995; 117(1): 27-40.

van der Helm FCT. A standardized protocol for motion recordings of the shoulder. Proceedings of the First Conference of the ISG. Delft 1997. p. 7-12.

Vermeulen HM, Stokdijk M, Eilers PH, Meskers CG, Rozing PM, Vliet Vlieland TP. Measurement of three dimensional shoulder movement patterns with an electromagnetic tracking device in patients with a frozen shoulder. Annals of the Rheumatic Diseases 2002; 61(2): 115-20.

Wang $\mathrm{CH}$, McClure P, Pratt NE, Nobilini R. Stretching and strengthening exercises: their effect on three-dimensional scapular kinematics. Archives of Physical Medicine and Rehabilitation 1999; 80(8): 923-9.

Woltring HJ, Huiskes R, de Lange A, and Veldpaus FE. Finite centroid and helical axis estimation from noisy landmark measurements in the study of human joint kinematics. Journal of Biomechanics, 1985; 18(5): 379-89.

Wu G, van der Helm FC, Veeger HE, Makhsous M, Van Roy P, Anglin C, Nagels J, Karduna AR, McQuade K, Wang X, Werner FW, Buchholz B. ISB recommendation on definitions of joint coordinate systems of various joints for the reporting of human joint motion-Part II: shoulder, elbow, wrist and hand. Journal of Biomechanics 2005; 38(5): 981-92.

Yano Y, Hamada J, Tamai K, Yoshizaki K, Sahara R, Fujiwara T, Nohara Y. Different scapular kinematics in healthy subjects during arm elevation and lowering: glenohumeral and scapulothoracic patterns. Journal of Shoulder and Elbow Surgery 2010; 19(2): 209-15. 


\section{FIGURES AND TABLES}

\section{Figure 1:}

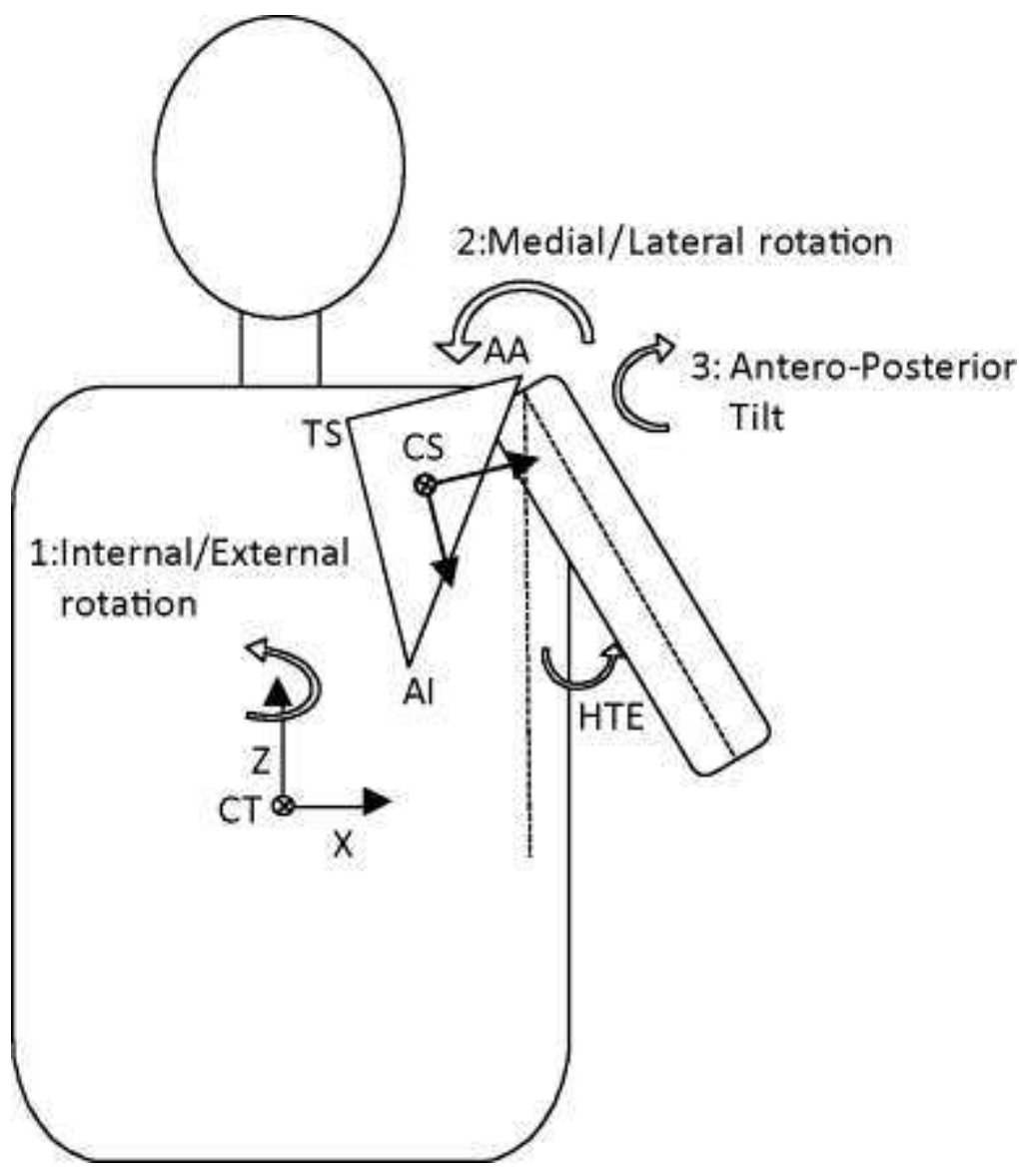

Schema of the body segments and corresponding reference frames for the thorax and scapula. Bony landmarks on the scapula: acromial angle (AA: angulus acromialis), root of the spine (TS: trigonum spinae scapulae) and inferior angle (AI: angulus inferior). CS is the barycentre of the scapula and CT the barycentre of the trunk. The arrows show the rotations as Euler angles in the following order: 1: Internal/External rotation, 2: Medial/Lateral/rotation, 3: Antero-Posterior Tilt. HTE : humero-thoracic elevation. 


\section{Figure 2:}
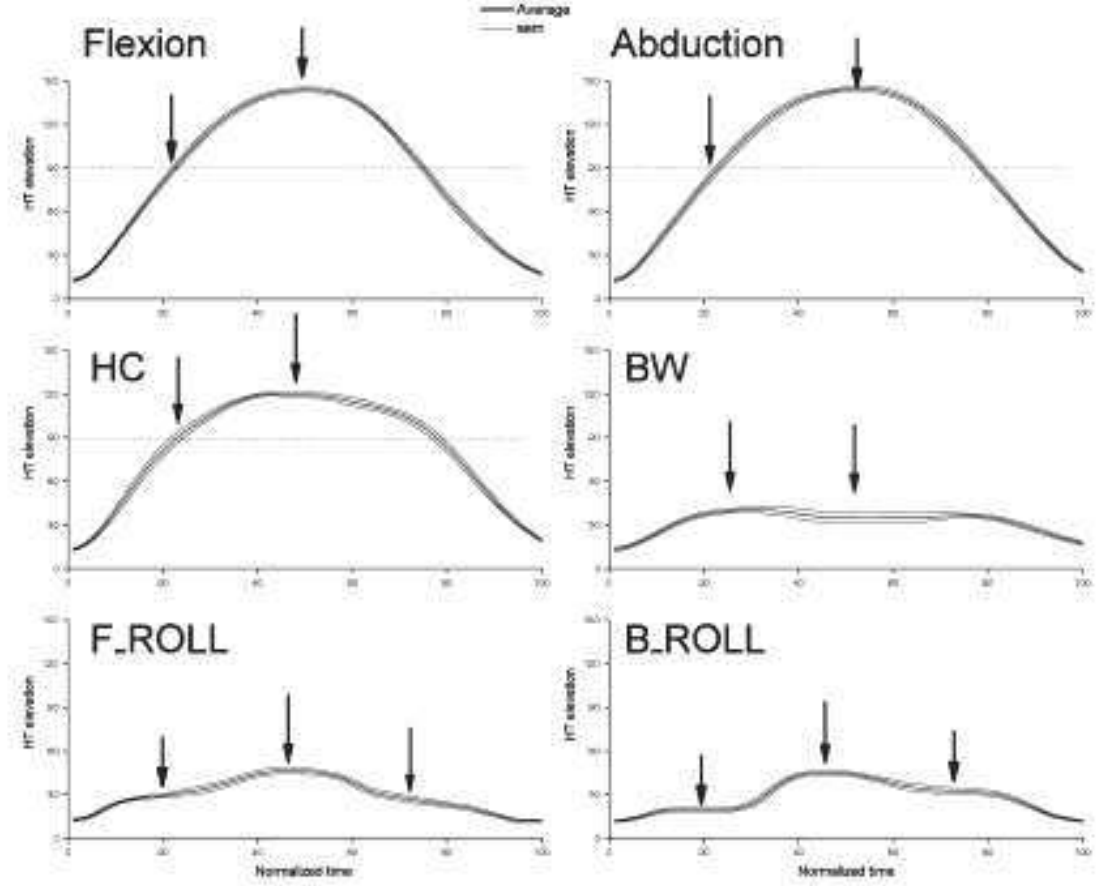

Time normalized averages of arm elevation relative to the thorax during the different tasks (between $\mathrm{t} 0$ and $\mathrm{tf}$ ). Each trace represents the average of the two successive trials in the group of subjects, right and left sides are averaged: Flexion: maximum elevation in the sagittal plane, Abduction: maximum elevation in the frontal plane HC: simulated hair combing: BW: simulated back washing; F-ROLL: forward rolling; B-ROLL backward rolling. Thick lines indicate the means and thin lines \pm one standard error of the mean (sem). The arrows indicate the time points analysed: $\mathrm{t} 1$ to $\mathrm{t} 3$. 
Figure 3:

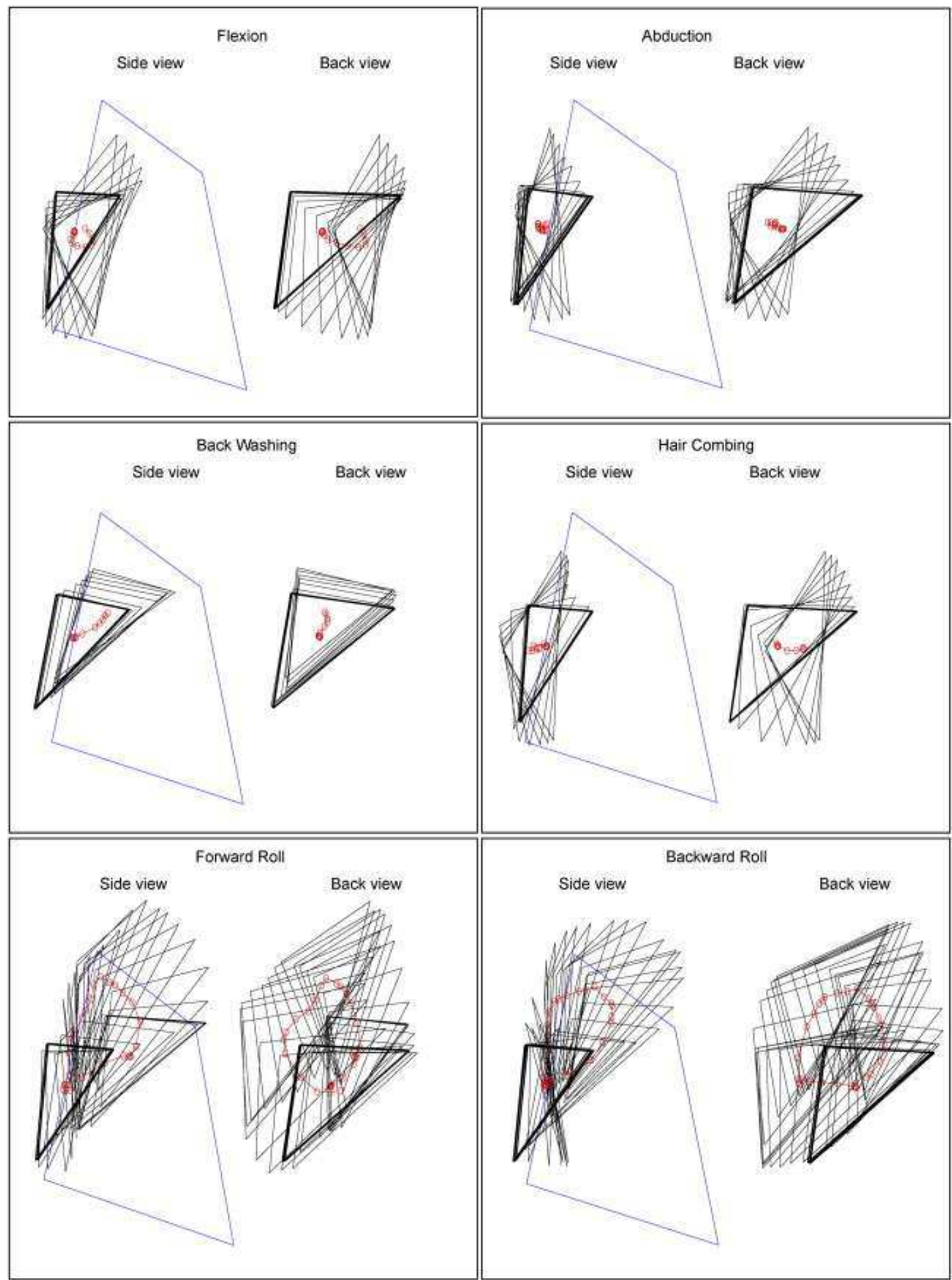

Examples of scapular kinematics during the six tasks in a representative subject. The blue polygon on the side view represents the initial posture of the thorax. Each triangle represents the scapula at a given time (from t0 to $\mathrm{t} 3$ for Flexion, Abduction, $\mathrm{HC}, \mathrm{BW}$ and from t0 to $\mathrm{t} 4 \mathrm{for} \mathrm{F}$ Roll and B-Roll). CS is represented by a (red) cross. The thick triangle indicates the initial posture. For the sake of clarity, only every third sample has been represented $(\sim 0.1 \mathrm{~s})$. 
Figure 4:

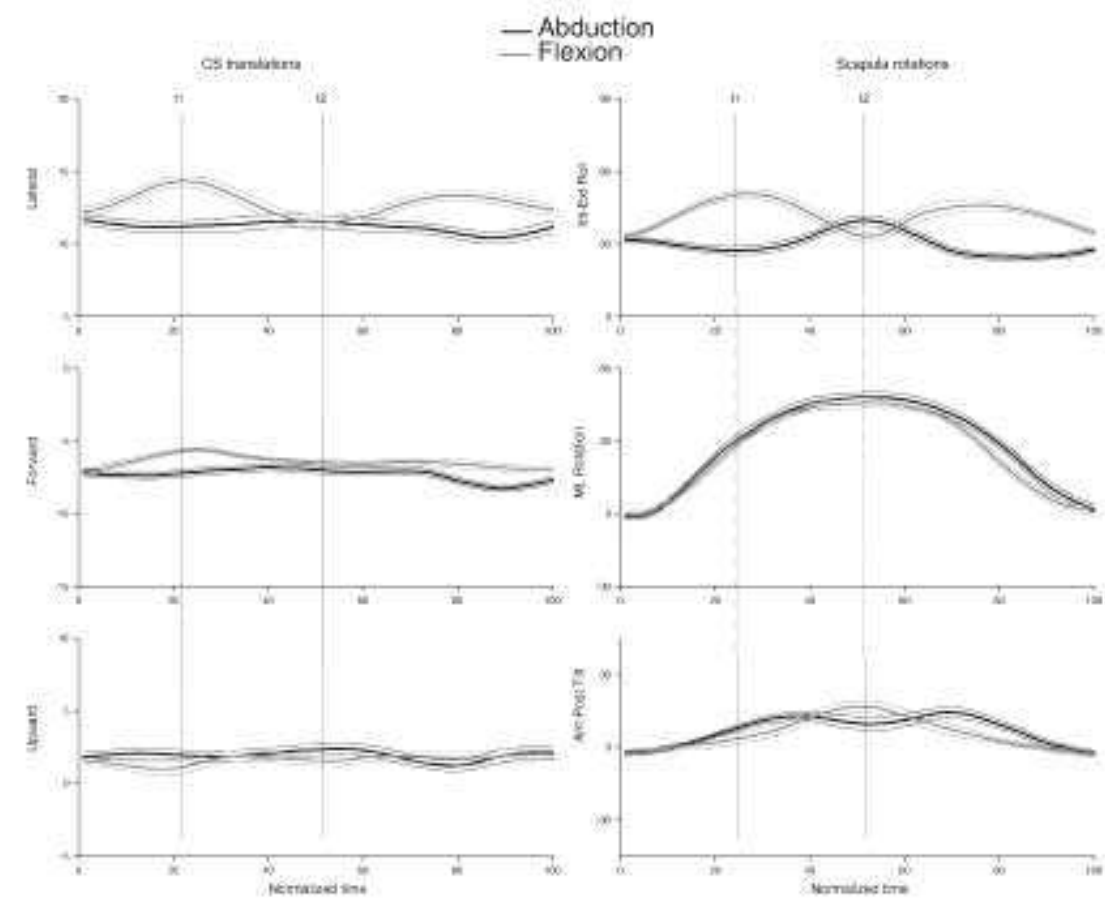

Time normalized averages of the coordinates of the centre of the scapula (CS) and scapular rotations during Flexion (grey lines) and Abduction (black lines). Same legend as Figure 2. 


\section{Figure 5:}

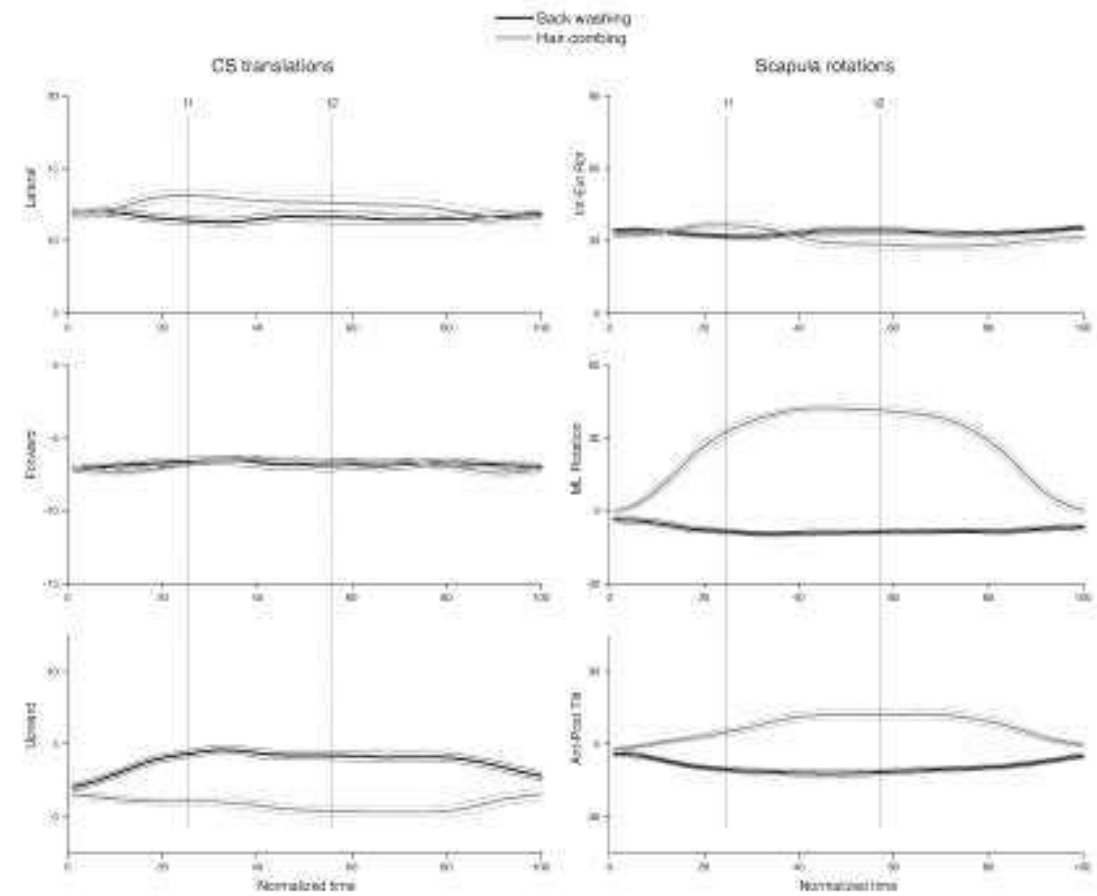

Time normalized averages of the coordinates of the centre of the scapula (CS) and scapular rotations during simulated hair combing (HC, grey lines) and back washing (BW, black lines). Same legend as Figure 2. 


\section{Figure 6:}

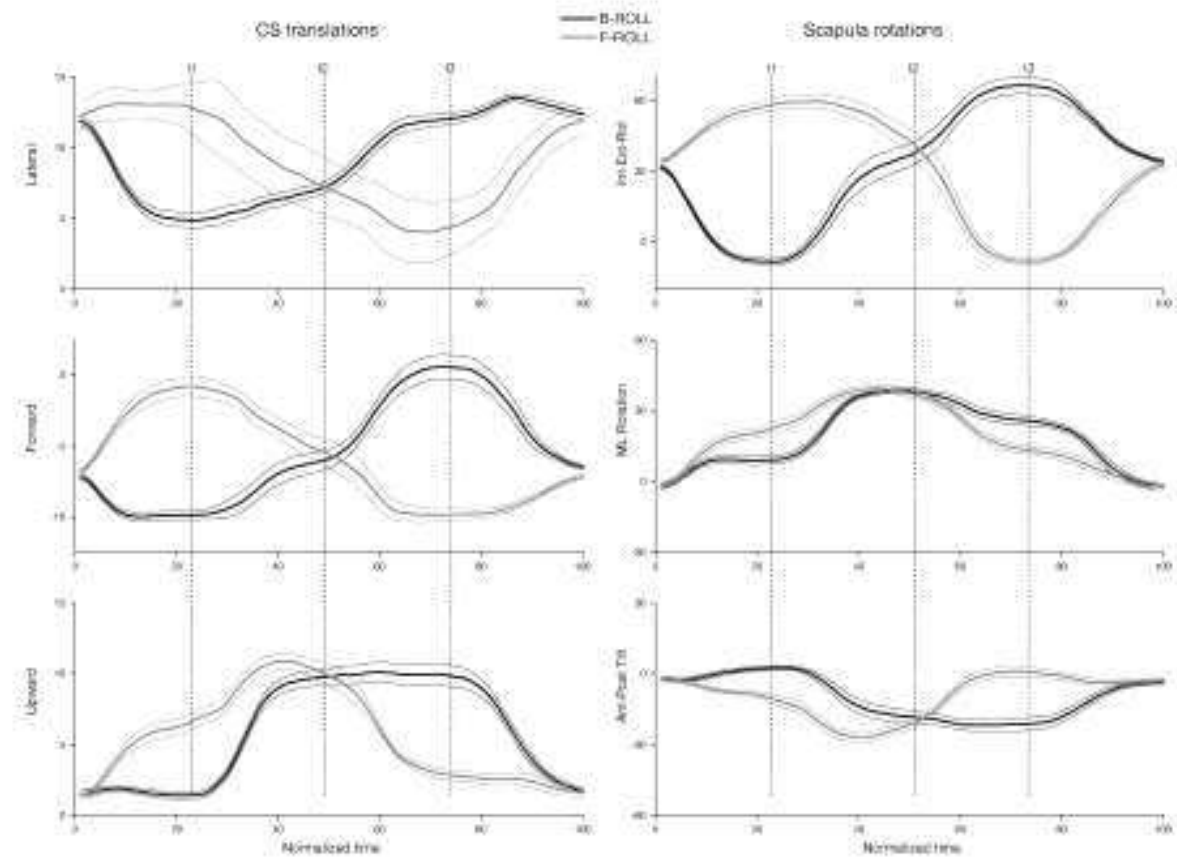

Time normalized averages of the coordinates of the centre of the scapula (CS) and scapular rotations during forward (F-ROLL, grey lines) and backward rolling (B-ROLL, black lines). Same legend as Figure 2. 


\section{Table 1.}

Table 1.

Amount of scapula rotation and displacement of its barycentre (CS) for planar arm elevation and simulated activities.

\begin{tabular}{|c|c|c|c|c|c|c|c|c|}
\hline \multirow{2}{*}{$\begin{array}{l}\text { Arm motion } \\
\text { task } \\
\text { Time-point }\end{array}$} & \multicolumn{2}{|l|}{ Abduction } & \multicolumn{2}{|l|}{ Flexion } & \multicolumn{2}{|l|}{$\mathrm{HC}$} & \multicolumn{2}{|l|}{ BW } \\
\hline & $t 1$ & $t 2$ & $t 1$ & $t 2$ & $t 1$ & $t 2$ & $t 1$ & $t 2$ \\
\hline \multicolumn{9}{|c|}{ Scapular rotations $\left(^{\circ}\right)$} \\
\hline $\begin{array}{l}\text { Internal/Extern } \\
\text { al rotation }\end{array}$ & $\begin{array}{l}-4.06 \pm 1 \\
07\end{array}$ & $n s$ & $\begin{array}{l}16.86 \pm 0 . \\
78\end{array}$ & $\begin{array}{l}17.23 \pm 0 \\
83\end{array}$ & $4.92 \pm 1$ & $\mathrm{~ns}$ & $\begin{array}{l}-2.8 \pm 0.5 \\
2\end{array}$ & ns \\
\hline $\begin{array}{l}\text { Medial/Lateral } \\
\text { rotation }\end{array}$ & $\begin{array}{l}29.64 \pm 1 . \\
6\end{array}$ & $\begin{array}{l}41.50 \pm 1 . \\
9\end{array}$ & $\begin{array}{l}27.06 \pm 1 . \\
24\end{array}$ & $\begin{array}{l}39.68 \pm 1 . \\
33\end{array}$ & $\begin{array}{l}32.03 \pm 1 \\
05\end{array}$ & $\begin{array}{l}28.34 \pm 3 . \\
64\end{array}$ & $\begin{array}{l}-6.11 \pm 0 . \\
47\end{array}$ & $\begin{array}{l}-4.58 \pm 0 . \\
76\end{array}$ \\
\hline $\begin{array}{l}\text { Anterior/posteri } \\
\text { or tilt }\end{array}$ & $\begin{array}{l}9.57 \pm 0.7 \\
9\end{array}$ & $\begin{array}{l}14.87 \pm 0 . \\
98\end{array}$ & $\begin{array}{l}5.02 \pm 1.3 \\
6\end{array}$ & $\begin{array}{l}8.59 \pm 1.6 \\
8\end{array}$ & $\begin{array}{l}6.36 \pm 0.8 \\
1\end{array}$ & $\begin{array}{l}7.80 \pm 1.2 \\
1\end{array}$ & $\begin{array}{l}-8.06 \pm 0 . \\
74\end{array}$ & $\begin{array}{l}-6.6 \pm 1.0 \\
7\end{array}$ \\
\hline \multicolumn{9}{|c|}{ Translations of the barycentre CS (mm) } \\
\hline Medial/Lateral & $-4.2 \pm 1.2$ & $n s$ & $24.5 \pm 1.8$ & $16.8 \pm 2.0$ & $12.6 \pm 1.8$ & $7.5 \pm 1.7$ & $-8.7 \pm 1.2$ & ns \\
\hline $\begin{array}{l}\text { Forward/backw } \\
\text { ard }\end{array}$ & ns & $n s$ & $16.1 \pm 2.1$ & $14.0 \pm 2.3$ & ns & $5.9 \pm 1.2$ & $5.8 \pm 1.3$ & ns \\
\hline $\begin{array}{l}\text { Upward/down } \\
\text { ward }\end{array}$ & ns & $n s$ & $\mathrm{~ns}$ & $n s$ & ns & ns & $27.6 \pm 1.9$ & $18.1 \pm 2.4$ \\
\hline 3D Amplitude & $10.0 \pm 1.1$ & $13.1 \pm 1.6$ & $\begin{array}{l}31.8 \pm 11 . \\
2\end{array}$ & $25.2 \pm 1.8$ & $30.2 \pm 1.8$ & $20.3 \pm 2.2$ & $16.4 \pm 1.6$ & $12.6 \pm 1.6$ \\
\hline
\end{tabular}

Values are mean differences \pm sem of scapular rotation (in degrees) and displacement of its barycentre (CS) (in millimeters) measured between the initial posture (at $t 0)$ and times $t 1$ and $t 2$ during the following tasks : Abduction, Flexion, simulated back washing $(\mathrm{BW})$ and hair combing $(\mathrm{HC})$. The time-point $\|$ corresponds to the first part of movement (elevation $\leq 90^{\circ}$ ) for Abduction, Flexion and $\mathrm{HC}$ and to the first local elevation maxima for BW. The timepoint 2 to the time of target posture (maximal arm elevation for Abduction, Flexion and $\mathrm{HC}$ or to the middle plateau for BW). Bold characters indicate $p<.001$, non-bold indicate $p<.05$. ns: non significant difference. Italic $s$ indicate values obtained for arm elevation $>120^{\circ}$. 


\section{Table 2}

Table 2.

Amount of scapula rotation and displacement of its barycentre (CS) for forward and backward rolling

Arm motion task

F-Roll

Time-point

$t 1$

$12 \quad t 3$

B-Roll

Scapular rotations $\left({ }^{\circ}\right)$

Internal/External

$22.36 \pm 1.68$

$21.10 \pm 2.72$

t3

$t 1$

t2

t3

rotation

$21.34 \pm 1.49$

$39.67 \pm 1.76$

Medial/Lateral rotation

$-7.02 \pm 1.69$

$39.67 \pm 1.76$

$-43.51 \pm 1.18$

$-36.91 \pm 1.74$

$8.27 \pm 3.01$

$39.36 \pm 2.59$

Anterior/posterior tilt

$-25.62 \pm 1.92$

$15.59 \pm 1.06$

$11.03 \pm 1.23$

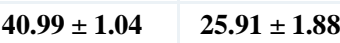

Translations of the barycentre CS $(\mathrm{mm})$

Medial/Lateral

$\mathbf{1 1 . 1} \pm \mathbf{1 . 9} \quad-37.9 \pm 3.8$

$3.73 \pm 0.67$

$5 \pm 0.39$

$-17.19 \pm 2.08$

$-19.48 \pm 1.79$

Forward/backward

$\mathbf{5 8 . 7} \pm \mathbf{4 . 7} \quad 27.4 \pm 5.2$

$49.2 \pm 5.1 \quad 96.8 \pm 6.8$

$-84.4 \pm 3.3$

$-65.5 \pm 3.7$

$-41.7 \pm 3.4$

$8.5 \pm 4.2$

Upward/downward

$77.8 \pm 6.8$

$108.9 \pm 7.9$

$-33.1 \pm 2.3$

$-24.4 \pm 3.0$

$17.0 \pm 4.6$

$86.3 \pm 6.0$

3D Amplitude

$13.3 \pm 3.2$

$-1.8 \pm 2.0$

$87.2 \pm 6.7$

$86.3 \pm 6.9$

Values are mean differences \pm sem of scapular rotation (in degrees) and translation of its barycentre (CS) (in millimeters) measured between the initial posture (at $t 0)$ and time-points $t 1, t 2$ and $B$ during forward rolling ( $F$-Roll) and backward rolling (B-Roll). The time-points $t 1$ to $B$ indicate the three main phases of the rolling movement. Bold characters indicate $p<.001$, non-bold indicate $p<.05$. 